\title{
genetics
}

\section{Restriction enzyme-generated siRNA (REGS) vectors and libraries}

\author{
George Sen $^{1,2,4}$, Tom S Wehrman ${ }^{1,2,4}$, Jason W Myers ${ }^{1} \&$ Helen M Blau $^{1-3}$
}

\begin{abstract}
Small interfering RNA (siRNA) technology facilitates the study of loss of gene function in mammalian cells and animal models, but generating multiple siRNA vectors using oligonucleotides is slow, inefficient and costly. Here we describe a new, enzyme-mediated method for generating numerous functional siRNA constructs from any gene of interest or pool of genes. To test our restriction enzyme-generated siRNA (REGS) system, we silenced a transgene and two endogenous genes and obtained the predicted phenotypes. REGS generated on average 34 unique siRNAs per kilobase of sequence. REGS enabled us to create enzymatically a complex siRNA library $\left(>4 \times 10^{5}\right.$ clones) from double-stranded cDNA encompassing known and unknown genes with $96 \%$ of the clones containing inserts of the appropriate size.
\end{abstract}

RNA interference technology provides a rapid means for assessing the effects of loss of function of any gene ${ }^{1-5}$. RNA interference specifically reduces a single mRNA species by introducing its corresponding double-stranded RNA (dsRNA). Application of the technology was initially limited to Drosophila melanogaster and Caenorhabditis elegans, because long dsRNA ( $>29$ nucleotides, nt) induces an interferon response and a subsequent nonspecific inhibition of mRNA translation in most mammalian cell types ${ }^{6,7}$. In $D$. melanogaster, long dsRNAs are cleaved to produce small dsRNA molecules of 21-23 nt (siRNAs) that are the effectors of gene silencing ${ }^{8}$. Transfection of siRNAs of this length in mammalian cells can circumvent the interferon response and efficiently target specific mRNAs for elimination ${ }^{7}$, but this effect is transient as the transfected siRNA is lost by degradation or diluted by cell division. To overcome this limitation, plasmid vectors encoding short hairpin RNAs with structures similar to active siRNA molecules ${ }^{9-15}$ were designed. The continuous production of these transcripts allows long-term silencing of genes by siRNA.

The successful production of siRNAs by plasmid-based methods encouraged the development of several vector types and delivery systems ${ }^{16-18}$. But several factors currently limit the use of DNA-derived siRNAs in mammalian cells. DNA-encoded siRNAs are sequencespecific and have a palindromic hairpin structure. As a result, siRNA vectors for a given gene must be constructed individually using sequence-specific oligonucleotide primer pairs. A recent report ${ }^{19}$ suggests that use of siRNA vectors can result in the modulation of nontargeted genes, and so isolation of multiple effective siRNA sequences is crucial to control for nonspecific off-target effects. Because only $25 \%$ of selected sequences are functional (for reasons not yet known), several constructs must be synthesized and cloned for each silenced gene. Thus, targeting every gene in the human genome would require hundreds of thousands of individually designed siRNA vectors; even then, only known genes would be represented in such a library.

Here we describe a system, called REGS, for readily generating multiple siRNA vectors from any gene or pool of double-stranded cDNAs. The process uses restriction enzymes to digest double-stranded cDNAs into multiple fragments and limit their size to $21 \mathrm{nt}$, small enough to avoid the interferon response. The resulting fragments are made into palindromic structures using hairpin loops and isothermal rolling circle amplification ${ }^{20}$ (RCA) and then cloned into expression vectors. The fidelity is high, as $96 \%$ of the inserts generated contained the expected 21-nt palindromic sequence. REGS is effective, as shown by silencing of three distinct genes, encoding GFP, Oct-3/4 and MyoD. REGS obviates the need to select and synthesize specific oligonucleotides to silence each gene of interest, because the same set of oligonucleotides can be used for all genes. This enzyme-mediated process generated siRNAs of the requisite size to known and unknown genes and allowed us to create the first highly complex siRNA library.

\section{RESULTS}

\section{The REGS process}

The procedure for generating numerous unique siRNA vectors from double-stranded cDNAs is outlined in Figure 1. This stepwise process is validated and described in detail for the glucocorticoid receptor. To generate a large number of fragments per gene that could be cloned as siRNAs, we chose specific restriction enzymes that cut frequently and left identical overhangs (Step 1; Fig. 1). We ligated the fragments to a hairpin DNA oligonucleotide, the $3^{\prime}$ loop, containing an overhang compatible with the digested fragments (Step 2; Fig. 1). The 3' loop links the sense and antisense strands of the gene fragments so that the hairpin can be opened and the complementary strand synthesized.

${ }^{1}$ Department of Molecular Pharmacology, ${ }^{2}$ Baxter Laboratory in Genetic Pharmacology and ${ }^{3}$ Department of Microbiology and Immunology, 269 Campus Drive, CCSR 4225A Stanford University School of Medicine, Stanford, California 94305, USA. ${ }^{4}$ These authors contributed equally to this work. Correspondence should be addressed to H.M.B. (hblau@stanford.edu). 
Only fragments of the appropriate size (19-29 nucleotides) encode functional siRNAs. The fragments ligated to the $3^{\prime}$ loop differed markedly in size (Fig. 2a), with most fragments exceeding 29 nt, rendering them incompatible with siRNA expression in mammalian cells. To overcome this problem and generate clonable fragments of both suitable size and greater number, we engineered a restriction enzyme site (MmeI; ref. 21) to overlap the ligation site of the $3^{\prime}$ loop. Ligation of this loop to the gene fragments formed the complete enzyme recognition site for MmeI. MmeI cuts at precisely $20 \mathrm{nt} 3^{\prime}$ from its recognition sequence, generating fragments of $21 \mathrm{nt}$ (Step 2; Fig. 1). Digestion of the ligation product with $\mathrm{MmeI}$ generated a band of $34 \mathrm{nt}$, including 21 nt of gene-specific sequence ligated to the 13-nt 3' loop (Fig. 2a). Very few fragments of the appropriate size were generated using only the initial restriction enzyme cocktail (Step 1; Fig. 1). Thus, MmeI is essential to obtaining multiple functional siRNAs of the requisite size.

To generate a functional siRNA, the product of the MmeI digestion had to be denatured and the complementary strand synthesized. To this end, we engineered priming sites to facilitate the synthesis of the complementary strand into a hairpin oligonucleotide, the $5^{\prime}$ loop, which we ligated to the 2-nt overhang left by MmeI digestion (Step 3; Fig. 1). Although the $5^{\prime}$ loop ligates to itself (Fig. 2b), it also ligates efficiently to the $3^{\prime}$ loop plus 21-nt fragment, as indicated by appearance of a 60-nt band (Step 3; Figs. 1 and $\mathbf{2 b}$ ), yielding a circular structure.

Synthesis of the complementary strand was not feasible by PCR because of the stability of the central double-stranded region. To overcome this problem, we used a strand-displacing enzyme, $\Phi 29$ DNA polymerase, (Step 4; Fig. 1). When primer RCA1, specific to the $5^{\prime}$ loop, is added to the circular structure, $\Phi 29$ is primed, disrupting the hairpin structure and allowing synthesis of the complementary strand. This process is continuous so that the enzyme repeatedly replicates the DNA around the dumbbell, displacing the newly synthesized strand after each cycle and amplifying the DNA by RCA. The result is a long, single-stranded DNA concatemer. Because the RCA2 primer is included in the reaction, the complementary strand is concurrently amplified, creating a doublestranded DNA concatemer.

To isolate the final DNA products with the appropriate structure, we digested the double-stranded concatemers resulting from the RCA reaction with BglII and MlyI (Step 5; Fig. 1), yielding an 80-nt fragment encoding the clonable siRNA sequence (Fig. 2c). To allow ligation of the BglII-MlyI-digested product, we modified the original pSuper retroviral vector to make the $3^{\prime}$ cloning site blunt-ended immediately before the RNA polymerase III termination site TTTTTGGAA; we designated this vector vREGS. Transcription of the siRNA is initiated by the $\mathrm{H} 1$ promoter directly upstream of the $B g l I I$ site and terminated at the poly-T region in the termination signal. Insertion of the digested 80 -nt REGS products into vREGS yielded the desired product devoid of extraneous sequences at either end of the transcribed siRNA (Step 5; Fig. 1). But this product still included excess $3^{\prime}$ loop. We had intentionally elongated the $3^{\prime}$ loop to
Figure 1 The REGS process. Step 1: The genes to be silenced are fragmented using restriction enzymes (Hinpl, BsaHI, Acil, Hpall, HpyCHIV and $T a q \alpha I)$ whose recognition sites are abundant in the genome and result in the same 2-nt overhang (CG) to facilitate cloning. The purpose of this step is to generate as many siRNA constructs per gene as possible. Step 2: The fragments are ligated to an oligonucleotide, which forms a hairpin loop ( $3^{\prime}$ loop), to link the sense and antisense strands. The $3^{\prime}$ loop contains a sufficiently long double-stranded stretch to allow efficient self-annealing and ligation by T4 DNA ligase. Because the 3' loop sequence must be longer than that accommodated in a noninterferon-inducing transcribed siRNA, a $B a m H I$ restriction site was engineered into the $3^{\prime}$ loop to eliminate the extraneous sequence after the first cloning reaction (see Step 6 below). To limit the size of the gene-specific fragments that would be transcribed into siRNAs, a recognition sequence for the Mmel restriction enzyme, which cleaves exactly $20 \mathrm{nt}$ from its recognition site, was engineered into the $3^{\prime}$ loop. Thus, on cleavage with $\mathrm{Mmel}$, all fragments that were ligated to the $3^{\prime}$ loop are of functional size. Step 3: The $5^{\prime}$ hairpin loop contains two specific restriction sites essential to subsequent cloning into the expression vector. Ligation of the $5^{\prime}$ loop to the Mmel-digested product generates a singlestranded closed circular dumbbell structure. Step 4: RCA is used to amplify the product of the second ligation reaction and to create linear double-stranded DNA for cloning. The $\$ 29$ DNA polymerase used in RCA displaces the newly synthesized strand, allowing repeated replication. As a result, RCA of the ligation product yields a concatemer of palindromic double-stranded DNA encoding siRNA molecules. Step 5: Digestion with Bg/lI and Mlyl allows insertion into vREGS. Step 6: The plasmids are digested with BamHI to eliminate the extraneous sequence and then religated, forming the final product: expression-ready siRNA vectors. The transcribed product is shown at the bottom as a product of REGS in comparison with those obtained from conventional cloning into pSuper.
Step 1

Step 2

Step 3
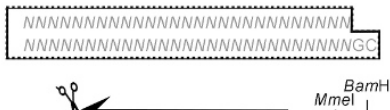

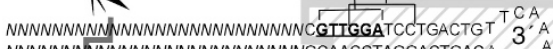

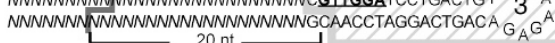
Mamel

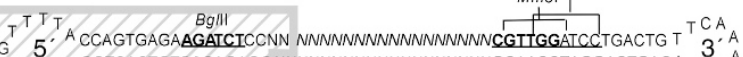

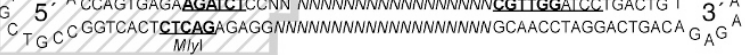

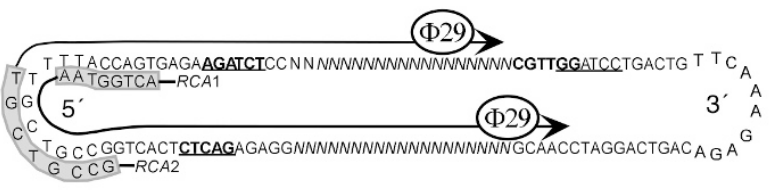

Step 5

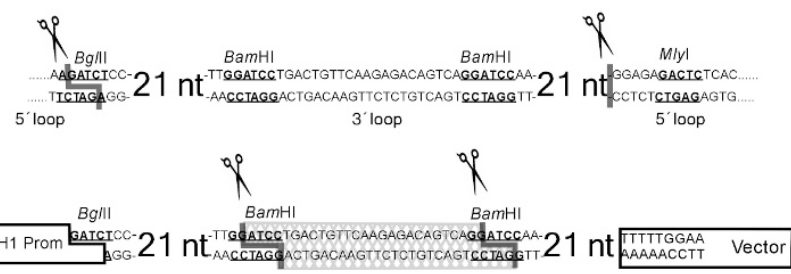

Final vector
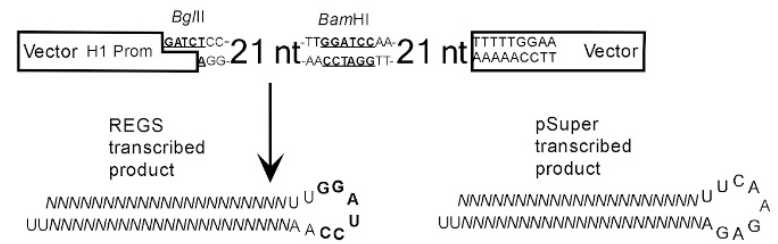

Transcribed 
Figure 2 Generation of multiple siRNA constructs using REGS. (a) Ligation of the $3^{\prime}$ loop to restriction enzyme-digested glucocorticoid receptor (GR) followed by Mmel digestion. Lane 7 shows the glucocorticoid receptor digested with the restriction enzymes Hinpl, BsaHI, Acil, Hpall, HpyCHIV and Taqol. The digested glucocorticoid receptor fragments were ligated to the $3^{\prime}$ loop, as shown by the upward shift in bands in lane 5 . Ligation of the $3^{\prime}$ loop to glucocorticoid receptor fragments followed by digestion with $\mathrm{Mmel}$ results in a band at $34 \mathrm{nt}$, which corresponds to the $3^{\prime}$ loop plus $21 \mathrm{nt}$ of glucocorticoid receptor sequence (lane 6). The predominant band of $\sim 30$ nt in lanes 4-6 is the $3^{\prime}$ loop self-ligated. (b) Ligation of the $5^{\prime}$ loop to the glucocorticoid receptor (GR) fragments plus the $3^{\prime}$ loop. The $5^{\prime}$ loop was self-ligated, forming a 45-nt band (lane 3). Ligation of the $5^{\prime}$ loop to the glucocorticoid receptor fragments plus the $3^{\prime}$ loop results in the desired 60-nt product (lane 4). (c) Generation of palindromic double-stranded DNA encoding siRNA molecules. RCA using primers towards the $5^{\prime}$ loop was done on all samples. Digestion with Bg/lI and $M / y l$ of the $5^{\prime}$ loop plus the glucocorticoid receptor fragments plus the $3^{\prime}$ loop shows the appearance of the expected 80-nt band (black arrowhead) containing the desired product and a 38-nt band containing the remnants of the $5^{\prime}$ loop (lane 7). Digestion with Bg/II and Mlyl of the self-ligated 5' loop results in the expected 38-nt band (lane 5).

ensure efficient self-annealing and ligation to the gene fragments by T4 DNA ligase (Step 2; Fig. 1). Digestion with BamHI removed the excess sequence, and religation yielded expression-ready siRNA vectors (Step 6; Fig. 1).

\section{Application of REGS to silencing single genes}

The only difference between the products of REGS and conventionally created siRNAs is the loop structure that connects the sense and antisense sequences. To test whether this loop (Fig. 1) affected siRNA function, we compared the previously published pSuper loop with the vREGS loop. We generated four 19-nt siRNAs to GFP with the pSuper loop and cloned them into pSuper Retro by traditional oligonucleotide synthesis. The sequence corresponding to $\mathrm{nt}$ 489-508 efficiently mediated silencing of GFP (data not shown). We then cloned the GFP siRNA sequence using the vREGS loop. We

Figure 3 Generation of multiple GFP

siRNA constructs and knock-down of GFP

expression. (a) Flow cytometric analysis of siRNA constructs targeting GFP. Primary myoblasts constitutively expressing GFP were transduced with siRNA constructs targeting GFP. vREGS was used as a negative control (blue) and the parental myoblasts show the autofluorescent baseline value (green). The upper panel compares the silencing efficiency between the same siRNA sequence targeting GFP cloned using the pSuper loop (red, pSuper 489) or the vREGS loop (purple, REGS GFP 489). The bottom panel shows four REGS constructs that knock down GFP expression to different degrees. (b) Westernblot analysis of GFP siRNA constructs. vREGS and an siRNA construct targeting Oct-3/4 (REGS Oct-792) were used as negative controls (lanes 1 and 2). pSuper 489 and REGS GFP 489 knocked down expression of GFP to a similar degree, indicating that the vREGS loop does not adversely affect gene silencing. The four REGS constructs that successfully silenced GFP by flow cytometry also show knock-down of GFP expression by western-blot analysis (lanes 5-8). The percentage of GFP knock-down was calculated by normalizing to the loading control, $\alpha$-tubulin. (c) GFP digested with restriction enzymes Hinpl, BsaHI, Acil, Hpall, $\mathrm{HpyCHIV}$ and Taq $\alpha$ I. The sequences of siRNA constructs isolated from GFP are shown in red. Cyan indicates the constructs that were possible but not isolated. Regions in green are sequences too far away from a restriction site or too short to be functional siRNAs. The numbered bars below the diagram show the extent of each siRNA that could be isolated and correspond to the numbered sequences in $\mathbf{d}$. (d) Frequencies of siRNA constructs targeting different regions of GFP. Twenty-six siRNA constructs against GFP can be generated; 18 of these were isolated, 9 antisense and 9 sense. Asterisks denote sequences that could silence GFP expression.

a
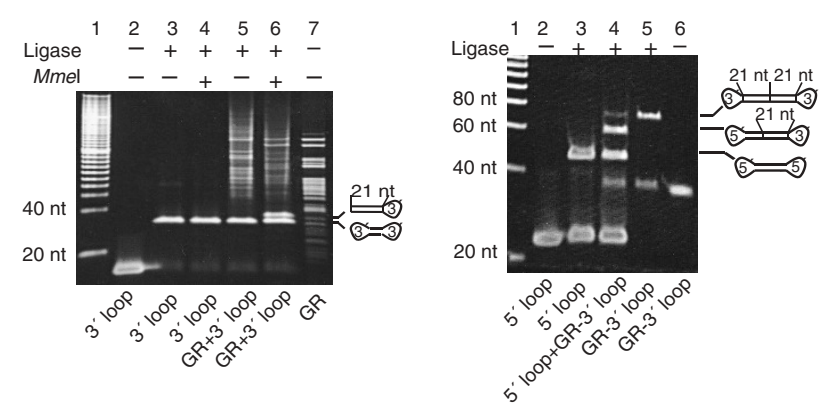

C

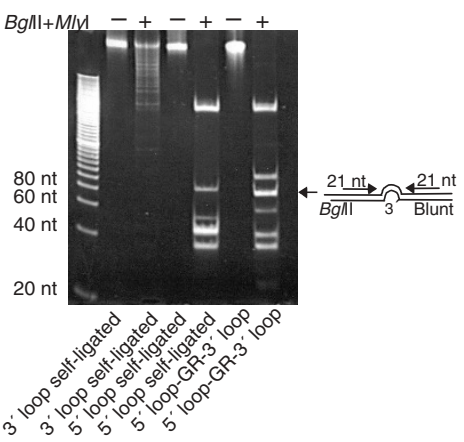

transfected both constructs into packaging cells and used the supernatants to infect primary myoblasts previously engineered to constitutively express GFP. The pSuper-GFP-489 and vREGS-GFP489 constructs both caused a $90 \%$ decrease in GFP fluorescence as analyzed by flow cytometry (Fig. 3a). Western-blot analysis showed $82 \%$ and $77 \%$ silencing of GFP by pSuper-GFP-489 and REGSGFP-489, respectively (Fig. 3b). Thus, the knockdown of GFP was essentially the same irrespective of loop structure. b

\section{Percent}

Knock-dow

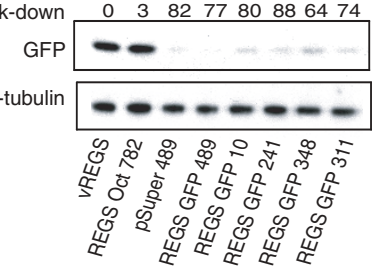

C

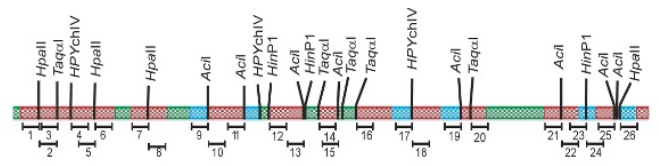

d

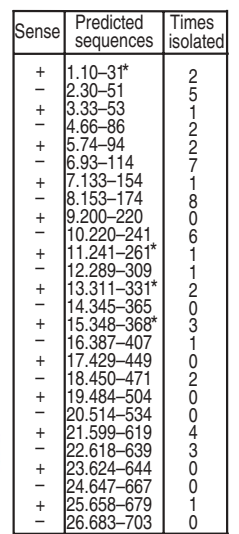


a

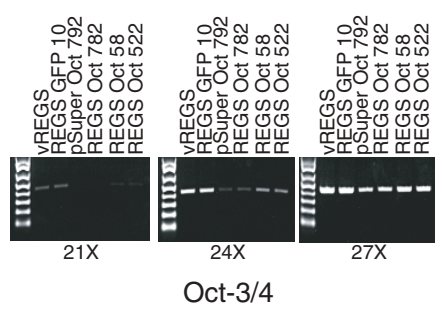

b
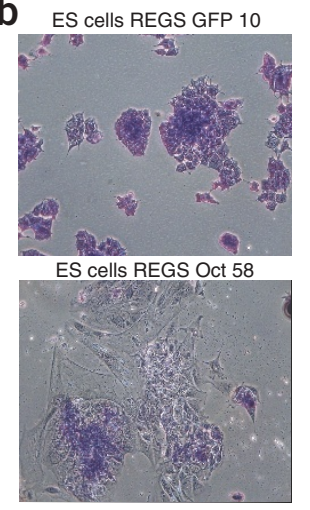

Violet precipitates: alkaline phosphatase expression
ES cells REGS Oct 782

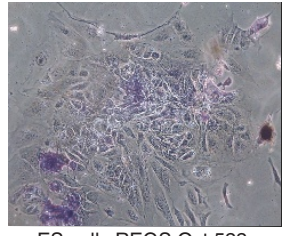

ES cells REGS Oct 522

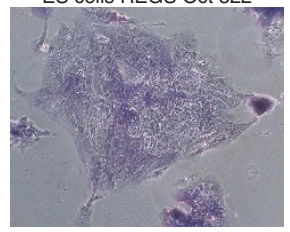

C

Figure 4 Generation of multiple siRNA constructs and silencing of Oct-3/4 expression. (a) Semiquantitative RT-PCR analysis of Oct-3/4 expression. siRNA constructs targeting Oct-3/4 were transduced into ES cells. Three REGS-derived constructs showed silencing of Oct-3/4 expression by semiquantitative PCR (lanes 4-6). pSuper Oct 792 was used as a positive control. vREGS and REGS GFP 10 were used as negative controls. (b) Knock-down of expression of Oct$3 / 4$ results in loss of alkaline phosphatase expression and differentiation of ES cells into trophoblasts. Cells transduced with REGS siRNAs Oct 58 , Oct 522 and Oct 782, which showed knock-down of expression of Oct-3/4 by RT-PCR, differentiated into trophoblasts, as shown by a large, flattened morphology and loss of alkaline phosphatase expression. Cells transduced with an irrelevant REGS siRNA (GFP 10) showed no trophoblast formation. (c) Knock-down of expression of Oct-3/4 causes downregulation of ES cell-specific genes ESG1 and UTF1 and upregulation of H19, a gene associated with differentiation by semiquantitative PCR. $\beta$-actin was used as an internal control in a (data not shown) and $\mathbf{c}$.

To determine the representation of the possible products from a single gene, we carried out the REGS procedure on GFP and analyzed 52 resulting clones (Fig. 3c). From the 52 sequenced plasmids, we obtained 18 unique siRNA retroviral constructs for GFP of the 26 possible (Fig. 3d). REGS facilitates the cloning of sense- and antisense-oriented siRNAs with equal probability and, as expected, half of the 18 unique constructs were cloned with the 21 -nt sense-strand $5^{\prime}$ to the loop (sense orientation; Fig. 3d). Four of the nine sense constructs knocked down expression of GFP when transduced into primary myoblasts constitutively expressing GFP, whereas none of the antisense constructs were effective, consistent with previous reports $^{22}$. siRNAs 10-31 and 241-261 knocked down expression of

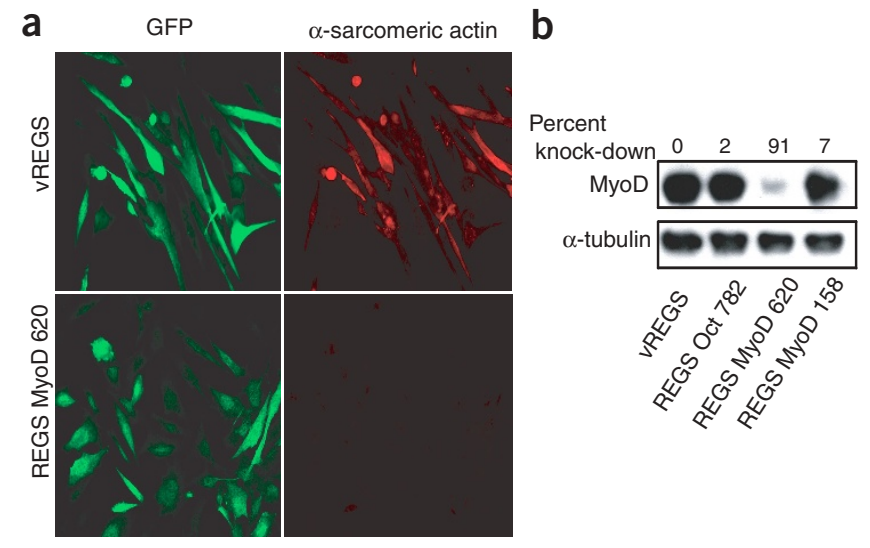

Figure 5 Knock-down of MyoD expression. (a) Silencing of MyoD expression blocks terminal differentiation of myoblasts. Primary myoblasts constitutively expressing GFP were transduced with REGS construct MyoD 620 or the negative control vREGS and cultured in differentiation medium for $2 \mathrm{~d}$. REGS MyoD 620 completely prevented differentiation of myoblasts to myotubes. Cells were also stained for $\alpha$-sarcomeric actin, a cytoskeletal protein found only in differentiated myotubes. (b) Western-blot analysis of MyoD knock-down using siRNA construct REGS MyoD 620.
GFP by nearly $90 \%$, as determined by flow cytometry, whereas GFP siRNAs 311-331 and 348-368 knocked down expression by $\sim 85 \%$ (Fig. 3a). Results from western-blot analysis (Fig. 3b) were consistent with the flow cytometry data.

\section{Knock-down of endogenous genes by REGS vectors}

We tested the efficacy of siRNA molecules generated by REGS to silence expression of Oct-3/4 in embryonic stem (ES) cells. Oct-3/4 is a transcription factor that is essential for the self-renewal of ES cells ${ }^{23}$. Reduction in Oct-3/4 expression results in the differentiation of ES cells to trophoblasts. Using REGS, we obtained six sense and five antisense constructs. Three of the sense-strand sequences, $58-78,522-541$ and 782-803, knocked down expression of Oct-3/4 (Fig. 4a). Oct siRNA 782-803 had the most pronounced knockdown effect, similar to that of Oct 792-811, which was previously constructed in pSuper Retro by traditional methods and shown to mediate silencing (data not shown). All three constructs caused the differentiation of ES cells to trophoblasts, as evidenced by large, flattened cell morphologies, and a subsequent loss of alkaline phosphatase staining (Fig. 4b). As expected, this change in phenotype was accompanied by the downregulation of expression of other proteins associated with ES cells, UTF1 (ref. 24) and ESG-1 (ref. 25). Conversely, expression of H19 (ref. 23), a marker for ES-cell differentiation, was highly upregulated (Fig. 4c).

We also tested REGS-mediated silencing of another endogenous gene, MyoD. MyoD is a basic helix-loop-helix transcription factor that is essential for the differentiation of myoblasts to myotubes ${ }^{26}$. We transduced primary myoblasts that constitutively expressed GFP with six sense siRNA constructs generated from MyoD using REGS. The siRNA corresponding to MyoD 620-640 completely blocked differentiation, as shown by the absence of myotube formation and $\alpha$ sarcomeric actin staining (Fig. 5a). The other five siRNA MyoD constructs had no effect. Western-blot analysis of these cells cultured in growth medium showed a $91 \%$ knock-down of MyoD expression by REGS MyoD 620-640, whereas another sense-strand construct, REGS MyoD 158 had little effect (Fig. 5b). 


\section{Construction of a REGS library}

The REGS process can not only produce large numbers of unique siRNA constructs simultaneously per gene, but can also generate sufficient numbers to yield an siRNA library that spans the entire transcriptome. To test this possibility, we carried out REGS on the inserts excised from a cDNA library. The cloning procedures were essentially identical to those described in Figures $\mathbf{1}$ and $\mathbf{2}$ for REGS, except at Step 4 , we carried out $20 \mathrm{RCA}$ reactions for $2 \mathrm{~h}$ to enhance the complexity of the library. The complexity of the library was estimated to be 415,000 independent clones, as determined by counting the number of colonies obtained from the first transformation (Step 5).

To validate the library, we isolated and sequenced 50 independent constructs. Of these, 48 constructs contained inserts with the appropriate structures and all were unique (Table 1). Forty-two of these clones had sequences identical to GenBank entries (Table 1), with approximately one-half cloned in the sense orientation. Three clones had no exact match in the mouse genome, and another three had contaminating sequences obtained from the cDNA library vector. Only two constructs contained no inserts. These results show that REGS can be used to generate a high-complexity library $(>4 \times$ $10^{5}$ ) in 4 days with $96 \%$ of the clones encoding siRNA inserts of the appropriate size.

\section{DISCUSSION}

We describe an enzyme-mediated method, REGS, for generating multiple siRNA constructs that target an individual gene or pool of cDNAs. By directly comparing an siRNA sequence that targeted GFP using the vREGS and pSuper vectors, we show that REGS-generated siRNAs are identical in form and function to traditionally created siRNA constructs. Furthermore, because the siRNAs can be produced in bulk from complex mixtures of DNA, libraries that encompass known and unknown genes can be created.

Many investigators report that 25\% of short hairpin RNA constructs are capable of suppressing the gene to which they are targeted. Our results are in agreement: on average, one of three sense-strand constructs silenced one of the three genes tested (GFP, four of nine constructs; Oct-3/4, three of six constructs; and MyoD, one of six constructs). A key advantage of REGS is the ability to generate a large number of unique siRNAs, increasing the likelihood of isolating multiple functional siRNA vectors for a given gene. This aspect of the technology is increasingly important in light of the recent finding ${ }^{19}$ that siRNA vectors can mediate unintentional sequence-specific silencing of nontargeted genes.

Efforts are underway to develop siRNA libraries that encompass every gene in the human genome $e^{6,27}$. The labor-intensive cloning process associated with generating at least four constructs for each gene using current methods is overwhelming. By contrast, using REGS, we were able to generate an siRNA library including approximately 415,000 inserts using a cloning process that requires only 4 days. As a single retroviral insertion is capable of silencing an overexpressed gene (Supplementary Fig. 1 online), a REGS library could be screened in bulk, using methods similar to those used for cDNA libraries. siRNA libraries generated from cDNA populations have the advantage of allowing the isolation of siRNA vectors that target unknown genes or differentially spliced transcripts.

Because the REGS-generated siRNA library is derived from an existing cDNA library, the complexity and bias of the siRNA library is dictated by the starting material. Thus, using a normalized or subtracted cDNA library will limit the biases inherited by the siRNA library. cDNA libraries are traditionally biased towards smaller genes, but the siRNA library is biased towards larger genes because the restriction enzymes used by REGS generate more fragments from longer DNA sequences. The yield of approximately 34 unique siRNA constructs per 1,000 nt is high, but there are some limitations. Half of the constructs will probably be nonfunctional owing to orientation, and one-third to one-fourth of sense-strand constructs are effective. As a result, one-sixth to one-eighth of all REGS vectors can be expected to mediate gene silencing. Given the large number of siRNAs generated using REGS, however, these limitations can be overcome.

Table 1 Sequences isolated from the REGS siRNA library

\begin{tabular}{|c|c|c|}
\hline siRNA location & Gene & $\begin{array}{c}\text { GenBank } \\
\text { accession number }\end{array}$ \\
\hline $2,966-2,985-$ & ATP7a & AK040753.1 \\
\hline $278-258-$ & Pleiotrophin & BC002064.1 \\
\hline $3,286-3,269-$ & RP23-263E3 & AC122295.4 \\
\hline $6,296-6,275-$ & Mitochondrial & V00711.1 \\
\hline $493-473-$ & GABA(A) receptor-associated protein 2 & NM_026693.2 \\
\hline $1,465-1,445-$ & IMAGE:4527839 Ubiquitin conj enz homolog & BC016203.1 \\
\hline $621-601-$ & Ferritin heavy chain & BC012314.1 \\
\hline $2,427-2,407-$ & IMAGE:6489956 & BC049362.1 \\
\hline $3,204-3,225+$ & Exportin 7 & BC029702.1 \\
\hline $805-785-$ & Bat5 & NM_178592.2 \\
\hline $415-435+$ & EEF-2 & BC002233.1 \\
\hline $10,533-10,553+$ & Y1 globin & $\mathrm{X} 14061.1$ \\
\hline $2,142-2,122-$ & $\mathrm{CDC} 46$ & D26090.1 \\
\hline $2,761-2,741-$ & Angiotensin converting enzyme & BC040404.1 \\
\hline $7,484-7,505+$ & Fatty acid synthase & BC046513.1 \\
\hline $1,549-1,529-$ & IMAGE:5355806 EST & BC024847.1 \\
\hline $450-471+$ & CaMKII-gamma & NM_178597.2 \\
\hline $739-760+$ & Idb3 & NM_008321.1 \\
\hline $1,581-1,601+$ & PI3K subunit p85 alpha & BC051106.1 \\
\hline $2,771-2,791+$ & EST IMAGE:3491837 & AC123871.4 \\
\hline $1,147-1,126-$ & IMAGE:4194837 & BC029813.1 \\
\hline $713-692-$ & Enolase 1 & BC024644.1 \\
\hline $1,138-1,158+$ & F-box only protein & ВС034854.1 \\
\hline $78-58-$ & Similar to ribosomal $\mathrm{L7a}$ & XM_193935.1 \\
\hline $951-972+$ & Proprotein convertase subtilisin/kexin type 5 & BC013068.1 \\
\hline $758-779+$ & Krt2-5 & NM_027011.1 \\
\hline $347-367+$ & Tubulin alpha 6 & BC022182.1 \\
\hline $265-244-$ & Pou $5 f 1$ & NM_013633.1 \\
\hline $488-509+$ & alpha-2-HS-glycoprotein & BC019822.1 \\
\hline $1,625-1,605-$ & Prolyl 4-hydroxylase, beta polypeptide & BC008549.1 \\
\hline $497-518+$ & Guanine nucleotide binding protein & BC048834.1 \\
\hline $3,588-3,567-$ & Ubiquitin specific protease 7 & BC046963.1 \\
\hline $768-748-$ & Dtprp & NM_010088.1 \\
\hline $2,263-2,284+$ & Similar to pol protein & XM_196572.2 \\
\hline $1,437-1,417-$ & Sept3 & AC104325.28 \\
\hline $891-871-$ & IMAGE:1515563 & AK014200.1 \\
\hline $2,002-2,022+$ & RIKEN cDNA C230075L19 & BC048924.1 \\
\hline $1,271-1,252-$ & Glutathione peroxidase 3 (Gpx3) & NM_008161.1 \\
\hline $2,003-2,024+$ & MLV-related provirus NL1 integrase & AY219562.2 \\
\hline $13-33+$ & Serum/glucocorticoid regulated kinase & BC005720.1 \\
\hline $1,044-1,064+$ & $\mathrm{Ca}^{2+}$-dependent ER nucleoside diphosphatase & BC020003.1 \\
\hline $2,824-2,846+$ & Similar to cyclin L ania-6a & BC023747.1 \\
\hline
\end{tabular}

We isolated and sequenced 50 clones from the original library. The position of the gene that matches the coding siRNA is indicated in the first column, with the symbol to the right indicating the orientation of the sequence in the vector $(+$, sense; -, antisense). Of the 50 sequences, 48 contained inserts of the proper size, 3 were from contaminating vector sequences and 3 had no identical matches in the GenBank database; 20 were cloned in the sense orientation and 22 in the antisense orientation. All sequences isolated were unique. 
The REGS technology allows the rapid generation of multiple siRNAs per gene to sequences that are not predetermined and are of appropriate size. The production of a complex siRNA library to both known and unknown genes is unprecedented and should be broadly useful in elucidating gene function.

\section{METHODS}

Amplification of genes used for REGS. We generated the open reading frames for the glucocorticoid receptor (2,268 nt), GFP (721 nt), MyoD (960 nt) and Oct-3/4 (1,324 nt) by PCR amplification. Primer sequences are available on request. The PCR cycle consisted of 30 cycles of $94^{\circ} \mathrm{C}$ for $1 \mathrm{~min}, 60^{\circ} \mathrm{C}$ for $1 \mathrm{~min}$ and $72^{\circ} \mathrm{C}$ for $1 \mathrm{~min}$, except for the glucocorticoid receptor, which was cycled at $94^{\circ} \mathrm{C}$ for $1 \mathrm{~min}, 53^{\circ} \mathrm{C}$ for $1 \mathrm{~min}$ and $72^{\circ} \mathrm{C}$ for $3 \mathrm{~min}$ for 30 cycles.

vREGS generation. We created a 425-nt stuffer sequence derived from the Oct$3 / 4$ open reading frame using a $5^{\prime}$ primer (REGS STUFF A) containing a BglII site and a $3^{\prime}$ primer (REGS STUFF B) containing HindIII and BbsI sites. Primer sequences are available on request. We used the primers to amplify the stuffer sequence from cDNA derived from ES cells. We cloned the product into the $B g l I I-H i n d I I I$ site of pSuper retroviral vector (Oligoengine) to create vREGS. To prepare the vector for siRNA insertion, we digested vREGS with BglII-BbsI. The BbsI site cuts 6 nt away, leaving the 4-nt 5' -TTTT-3' overhang. We used T4 DNA polymerase to fill in the overhangs left by $B b s \mathrm{I}$, forming a blunt end. We cloned the siRNA inserts into vREGS BglII-blunt, directly downstream of the $\mathrm{H} 1$ promoter and upstream of the termination signal.

The REGS process: Step 1. We digested $5 \mu$ g of double-stranded cDNA for each gene of interest with HinpI, BsaHI, AciI, HpaII, HpyCHIV and TaqoI (New England Biolabs) and purified them using Qiaex II beads (Qiagen). All six restriction enzymes leave a $5^{\prime} \mathrm{CG}$ overhang to allow subsequent ligation to the $3^{\prime}$ loop containing a compatible 5' CG overhang.

Step 2. We ligated $3 \mu \mathrm{g}$ of the digested gene fragments to $1.5 \mu \mathrm{g}$ (2:1 ratio) of the $3^{\prime}$ loop (5'-CGTTGGATCCCGGTTCAAGAGACCGGGATCCAA-3') for $1 \mathrm{~h}$ and heat-inactivated it at $65^{\circ} \mathrm{C}$ for $10 \mathrm{~min}$. We ordered the PAGE-purified loop oligonucleotides from Integrated DNA Technologies. The $3^{\prime}$ loop contained a partial MmeI restriction enzyme site of $5^{\prime}$-TCCAA- $3^{\prime}$. Ligation of the $3^{\prime}$ loop to the gene fragments formed the complete enzyme recognition site of $5^{\prime}$ TCCAAC- $3^{\prime}$. The first nucleotide of the gene sequence provided the last $\mathrm{C}$ of the MmeI site, because the initial gene fragments end in a CG overhang. After digestion with $\mathrm{MmeI}$, which cuts $20 \mathrm{nt}$ away, the last $\mathrm{C}$ of the MmeI site and the 20 -nt fragment generate $21 \mathrm{nt}$ of gene-specific sequence. For $\mathrm{MmeI}$ digestion, we diluted the ligation reaction threefold into $\mathrm{MmeI}$ buffer including S-adenosyl-methionine and the $\mathrm{MmeI}$ enzyme (NEB) for $1 \mathrm{~h}$. We separated the reaction on a $20 \%$ Tris-borate-EDTA Novex gel (Invitrogen), excised the $\sim 34$-nt band (gene fragment and $3^{\prime}$ loop), fragmented it into small pieces and placed it in $0.5 \mathrm{M}$ salt for $3-5 \mathrm{~h}$ at $50^{\circ} \mathrm{C}$. We used Qiaex II beads (Qiagen) to purify the DNA from the salt solution according to manufacturer's instructions.

Step 3. We ligated $1 \mu \mathrm{g}$ of the purified band to $500 \mathrm{ng}$ of $5^{\prime}$ loop (5'-GGAGAG ACTCACTGGCCGTCGTTTTACCAGTGAAGATCTCCNN-3'; 2:1 ratio) for $1.5 \mathrm{~h}$. We separated it on a $10 \%$ Tris-borate-EDTA Novex gel and gel-purified the band of $\sim 60 \mathrm{nt}$. The $5^{\prime}$ loop is predicted to form a 15 -nt stem loop ending in a $3^{\prime}$ $\mathrm{NN}$ extension that is compatible with the overhangs left by the MmeI digestion.

Step 4. We carried out RCA using the TempliPhi 100 amplification kit according to manufacturer's protocol (Amersham Biosciences), except we used primers RCA1 and RCA2 specific to the $5^{\prime}$ loop. Primer sequences are available on request. We incubated the RCA reaction at $30^{\circ} \mathrm{C}$ for $12 \mathrm{~h}$ and heat-inactivated it at $65^{\circ} \mathrm{C}$ for $10 \mathrm{~min}$.

Step 5. We diluted RCA products 1:2 into buffer 2 (NEB) containing BglII and MlyI. We isolated the desired fragment (of $80 \mathrm{nt}$ ) from a $10 \%$ Trisborate-EDTA gel. We ligated $30 \mathrm{ng}$ of the BglII-MlyI fragment to $90 \mathrm{ng}$ of vREGS (1:3 ratio) and transformed it into Stbl2 bacterial competent cells (Invitrogen). We scraped the resulting bacterial colonies and isolated the siRNA constructs using a mini prep kit (Qiagen).
Step 6. We digested the plasmids with BamHI, gel-purified them and self-ligated them to produce the final siRNA constructs. We picked individual colonies and isolated plasmids. We digested the constructs with BamHI before sequencing to prevent the formation of secondary structure caused by the palindromic nature of the cloned inserts.

REGS library. We isolated the double-stranded cDNA from a mouse embryonic retroviral library (Clontech) from the vector sequences by digestion with $S f i$ (New England Biolabs) and gel-purified it. The protocol is the same as used for the other genes, except as noted. We used $5 \mu \mathrm{g}$ of double-stranded cDNA as starting material for the first ligation and scaled all loop amounts accordingly. In Step 4 , we carried out $20 \mathrm{RCA}$ reactions at $30{ }^{\circ} \mathrm{C}$ for $2 \mathrm{~h}$. We counted the colonies resulting from completion of Step 5 to determine the complexity of the library. We used dilutions from $0.45 \mathrm{ng}, 0.9 \mathrm{ng}, 4.5 \mathrm{ng}$ and $9 \mathrm{ng}$ of vector DNA to determine the number of colonies yielded per microgram of vector DNA. We then used $1 \mu \mathrm{g}$ of vector DNA to generate the siRNA library.

Cell culture. We isolated primary myoblasts from adult FVBNJ mice and grew them in Dulbecco's modified Eagle medium with $20 \%$ fetal calf serum and basic fibroblast growth factor as previously described ${ }^{28}$. Differentiation assays were done by placing myoblasts in Dulbecco's modified Eagle medium with 5\% horse serum for $2 \mathrm{~d}$. We obtained embryonic stem cells, line D3, from the American Type Culture Collection and grew them in Knockout Dulbecco's modified Eagle medium (GIBCO), 15\% knockout serum (GIBCO) and Lif (ESGRO from Chemicon).

Stable cell line production. We transfected ecotropic phoenix cells (gift from Garry Nolan, Stanford University) with $1.6 \mu \mathrm{g}$ of each REGS siRNA construct. Transfections were done in 12-well plates using Lipofectamine 2000 (Invitrogen) according to the manufacturer's instructions. We collected viral supernatants $48 \mathrm{~h}$ after transfection and added polybrene $\left(5 \mu \mathrm{g} \mathrm{m}^{-1}\right)$. We placed these supernatants on target cells and centrifuged them for $30 \mathrm{~min}$ at $2,000 \mathrm{~g}$. We infected cells four times and selected them with puromycin $(1 \mu \mathrm{g}$ $\left.\mathrm{ml}^{-1}\right) 1 \mathrm{~d}$ after the last infection.

Generation of GFP-expressing primary myoblasts. We cloned GFP into the MFG retroviral vector and transduced it into adult FVBNJ primary myoblasts. We sorted and cloned individual cells using the Facstar cell sorter (Becton Dickinson). We then used one clone for all GFP experiments.

Western-blot analysis. We trypsinized cells, pelleted them by centrifugation and resuspended and lysed them in buffer containing $1 \%$ Nonidet P-40, $150 \mathrm{mM}$ $\mathrm{NaCl}, 50 \mathrm{mM}$ Tris (pH 8.0), $1 \mathrm{mM}$ EDTA, $0.1 \%$ SDS, $0.5 \%$ sodium deoxycholate and a protease inhibitor cocktail (Roche). We quantified samples using BioRad's protein assay according to the manufacturer's instructions. We loaded $1 \mu \mathrm{g}$ of total protein for each sample in the expression analysis for GFP and $\alpha$-tubulin and $5 \mu \mathrm{g}$ of total protein for expression analysis for MyoD. We separated samples on NuPAGE 4-12\% Bis-Tris gradient gels (Invitrogen) and transferred them to Immobilon-P membranes (Millipore) for immunoblotting. We used polyclonal rabbit antibody to GFP (Molecular Probes, A-11122) at a dilution of 1:6,000 and mouse antibodies to $\alpha$-tubulin (Sigma, T5168) and to MyoD (PharMingen, $554130)$ at a dilution of 1:1,000. We used horseradish peroxidase-conjugated, secondary goat antibodies to mouse (Zymed Laboratories, 81-6520) and to rabbit (Zymed Laboratories, 81-6120) at a dilution of 1:5,000. We detected blots using ECL (Amersham Biosciences) according to the manufacturer's protocol. We quantified signals using a Lumi-Imager (Mannheim Boehringer). We normalized the densitometric data obtained from the GFP or MyoD band to that of $\alpha$-tubulin. We set the densitometric data from the control to $100 \%$ and show all other data as a percentage of the control value.

RNA isolation and semiquantitative RT-PCR. We extracted total RNA from ES cells using the RNeasy mini kit (Qiagen). We reverse-transcribed $1 \mu \mathrm{g}$ of total RNA using the $1^{\text {st }}$ Strand cDNA Synthesis Kit for RT-PCR (Roche). We used $1 \mu \mathrm{l}$ of cDNA for amplification using the Titanium Taq PCR kit from Clontech. The PCR cycles for all reactions were $94{ }^{\circ} \mathrm{C}$ for $1 \mathrm{~min}, 60^{\circ} \mathrm{C}$ for $1 \mathrm{~min}$ and $72{ }^{\circ} \mathrm{C}$ for $1 \mathrm{~min}$; the number of cycles depended on the gene. Primer sequences for Oct-3/4, UTF1, ESG-1 and H19 are available on request. We purchased mouse $\beta$-actin primers from Stratagene (302110). We 
carried out semiquantitative RT-PCR for Oct-3/4 using 21, 24 and 27 cycles; for $\beta$-actin using 19, 21 and 23 cycles; for UTF1 using 25 and 27 cycles; for ESG1 using 21 and 23 cycles; and for H19 using 21 and 24 cycles. We visualized PCR products on $1 \%$ agarose gels stained with ethidium bromide.

Alkaline phosphatase staining and immunofluorescence. We fixed embryonic stem cells and stained them using the Alkaline Phosphatase staining kit (Sigma, 85L-2) according to the manufacturer's instructions. For immunofluorescence, we fixed cells in $4 \%$ paraformaldehyde for $5 \mathrm{~min}$ and blocked them in buffer containing $2.5 \%$ normal goat serum, $0.3 \%$ Triton X-100 and $2 \%$ bovine serum albu$\min$ for $30 \mathrm{~min}$. We used mouse antibody to $\alpha$-sarcomeric actin (Sigma, A-2172) at 1:200 dilution and rabbit antibody to GFP (Molecular Probes, A-11122) at 1:2,500 dilution. Secondary antibodies were Texas Red-conjugated goat antibody to mouse IgM (Jackson, 115-075-075; 1:1,000) and Alexa 488-conjugated goat antibody to rabbit (Molecular Probes, A-11034; 1:1,000).

Note: Supplementary information is available on the Nature Genetics website.

\section{ACKNOWLEDGMENTS}

We thank A. Palermo for statistical assistance and E. Moreno for technical support. This work was supported by a fellowship from the Howard Hughes Medical Institute to G.S., a Biotechnology training grant from the US National Institutes of Health to T.S.W., a training grant from the US National Institutes of Health to J.M., and grants from the US National Institutes of Health and support from the Baxter Foundation to H.M.B.

\section{COMPETING INTERESTS STATEMENT}

The authors declare that they have no competing financial interests.

Received 17 September; accepted 9 December 2003

Published online at http://www.nature.com/naturegenetics/

1. Lee, S.S. et al. A systematic RNAi screen identifies a critical role for mitochondria in C. elegans longevity. Nat. Genet. 33, 40-48 (2003).

2. Carthew, R.W. Gene silencing by double-stranded RNA. Curr. Opin. Cell Biol. 13 244-248 (2001).

3. Shi, Y. Mammalian RNAi for the masses. Trends Genet. 19, 9-12 (2003),

4. Fire, A. et al. Potent and specific genetic interference by double-stranded RNA in Caenorhabditis elegans. Nature 391, 806-811 (1998).

5. Kamath, R.S. et al. Systematic functional analysis of the Caenorhabditis elegans genome using RNAi. Nature 421, 231-237 (2003).

6. Dykxhoorn, D.M., Novina, C.D. \& Sharp, P.A. Killing the messenger: short RNAs that silence gene expression. Nat. Rev. Mol. Cell Biol. 4, 457-467 (2003).

7. Elbashir, S.M. et al. Duplexes of 21-nucleotide RNAs mediate RNA interference in cultured mammalian cells. Nature 411, 494-498 (2001).
8. Zamore, P.D., Tuschl, T., Sharp, P.A. \& Bartel, D.P. RNAi: double-stranded RNA directs the ATP-dependent cleavage of mRNA at 21 to 23 nucleotide intervals. Cell 101, 25-33 (2000).

9. Sui, G. et al. A DNA vector-based RNAi technology to suppress gene expression in mammalian cells. Proc. Natl. Acad. Sci. USA 99, 5515-5520 (2002).

10. Yu, J.Y., DeRuiter, S.L. \& Turner, D.L. RNA interference by expression of short-interfering RNAs and hairpin RNAs in mammalian cells. Proc. Natl. Acad. Sci. USA 99 6047-6052 (2002)

11. Paul, C.P., Good, P.D., Winer, I. \& Engelke, D.R. Effective expression of small interfering RNA in human cells. Nat. Biotechnol. 20, 505-508 (2002).

12. Brummelkamp, T.R., Bernards, R. \& Agami, R. A system for stable expression of short interfering RNAs in mammalian cells. Science 296, 550-553 (2002).

13. Lee, N.S. et al. Expression of small interfering RNAs targeted against HIV-1 rev transcripts in human cells. Nat. Biotechnol. 20, 500-505 (2002).

14. Miyagishi, M. \& Taira, K. U6 promoter-driven siRNAs with four uridine 3' overhangs efficiently suppress targeted gene expression in mammalian cells. Nat. Biotechnol. 20, 497-500 (2002).

15. Paddison, P.J., Caudy, A.A., Bernstein, E., Hannon, G.J. \& Conklin, D.S. Short hairpin RNAs (shRNAs) induce sequence-specific silencing in mammalian cells. Genes Dev. 16, 948-958 (2002).

16. Rubinson, D.A. et al. A lentivirus-based system to functionally silence genes in primary mammalian cells, stem cells and transgenic mice by RNA interference. Nat. Genet. 33, 401-406 (2003).

17. Brummelkamp, T.R., Bernards, R. \& Agami, R. Stable suppression of tumorigenicity by virus-mediated RNA interference. Cancer Cel/ 2, 243-247 (2002).

18. Matsukura, S., Jones, P.A. \& Takai, D. Establishment of conditional vectors for hairpin siRNA knockdowns. Nucleic Acids Res. 31, e77 (2003).

19. Jackson, A.L. et al. Expression profiling reveals off-target gene regulation by RNAi. Nat. Biotechnol. 21, 635-637 (2003).

20. Detter, J.C. et al. Isothermal strand-displacement amplification applications for highthroughput genomics. Genomics 80, 691-698 (2002).

21. Boyd, A.C., Charles, I.G., Keyte, J.W. \& Brammar, W.J. Isolation and computer-aided characterization of Mmel, a type II restriction endonuclease from Methylophilus methylotrophus. Nucleic Acids Res. 14, 5255-5274 (1986).

22. Czauderna, F. et al. Functional studies of the PI(3)-kinase signalling pathway employing synthetic and expressed siRNA. Nucleic Acids Res. 31, 670-682 (2003).

23. Niwa, H., Miyazaki, J. \& Smith, A.G. Quantitative expression of Oct-3/4 defines differentiation, dedifferentiation or self-renewal of ES cells. Nat. Genet. 24, 372-376 (2000).

24. Okuda, A. et al. UTF1, a novel transcriptional coactivator expressed in pluripotent embryonic stem cells and extra-embryonic cells. EMBO J. 17, 2019-2032 (1998).

25. Tanaka, T.S. et al. Gene expression profiling of embryo-derived stem cells reveals candidate genes associated with pluripotency and lineage specificity. Genome Res. 12, 1921-1928 (2002).

26. Cornelison, D.D., Olwin, B.B., Rudnicki, M.A. \& Wold, B.J. MyoD(-/-) satellite cells in single-fiber culture are differentiation defective and MRF4 deficient. Dev. Biol. 224, 122-137 (2000).

27. Hannon, G.J. RNA interference. Nature 418, 244-251 (2002).

28. Rando, T.A. \& Blau, H.M. Primary mouse myoblast purification, characterization, and transplantation for cell-mediated gene therapy. J. Cell. Biol. 125, 1275-1287 (1994). 